CRYSTALLOGRAPHIC COMMUNICATIONS

ISSN 2056-9890

\section{Synthesis, crystal structure, DFT calculations and Hirshfeld surface analysis of 2-(1-decyl-2-oxo- indolin-3-ylidene)propanedinitrile}

\author{
Ibtissam Rayni, ${ }^{a}$ Youness El Bakri, ${ }^{a, b *}$ Chin-Hung Lai, ${ }^{c, d}$ L'houssaine El Ghayati, ${ }^{a}$ \\ El Mokhtar Essassi ${ }^{\mathrm{a}}$ and Joel T. Mague ${ }^{\mathrm{e}}$
}

Received 3 December 2018

Accepted 5 December 2018

Edited by L. Van Meervelt, Katholieke Universiteit Leuven, Belgium

Keywords: crystal structure; $\pi$-stacking; indole; Hirshfeld surface analysis.

CCDC reference: 1883193

Supporting information: this article has supporting information at journals.iucr.org/e

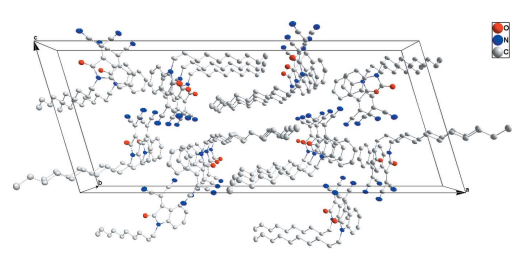

OPEN $\odot$ ACCESS
aLaboratoire de Chimie Organique Hétérocyclique, Centre de Recherche des Sciences des Médicaments, URAC 21, Pôle de Compétence Pharmacochimie, Av Ibn Battouta, BP 1014, Faculté des Sciences, Université Mohammed V, Rabat, Morocco, borganic Chemistry Department, Science Faculty, RUDN University, Miklukho-Maklaya st. 6, 117198 Moscow, Russian Federation, 'Department of Medical Applied Chemistry, Chung Shan Medical University, Taichung 40241, Taiwan, 'd Department of Medical Education, Chung Shan Medical University Hospital, 402 Taichung, Taiwan, and ${ }^{\mathbf{e}}$ Department of Chemistry, Tulane University, New Orleans, LA 70118, USA. *Correspondence e-mail: yns.elbakri@gmail.com

In the title molecule, $\mathrm{C}_{21} \mathrm{H}_{25} \mathrm{~N}_{3} \mathrm{O}$, the 1-decyl substituents are in an extended conformation and intercalate in the crystal packing to form hydrophobic bands. The packing is further organized by $\pi-\pi$-stacking interactions between pyrrole and phenyl rings [centroid-centroid distance $=3.6178(11) \AA$ ] and a

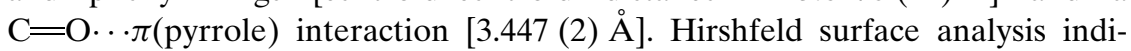
cates that the $\mathrm{H} \cdots \mathrm{N} / \mathrm{N} \cdots \mathrm{H}$ interactions make the highest contribution $(17.4 \%)$ to the crystal packing.

\section{Chemical context}

Knoevenagel condensation is a nucleophilic addition of an active hydrogen compound to a carbonyl group followed by dehydration in which a molecule of water is eliminated (Jones, 1967). The indole scaffold including isatin ( $1 H$-indole-2,3dione) represents an important structural subunit for the discovery of new drug candidates (Pandeya et al., 2005). The carbonyl group in the 3-position of isatin is known to be active in various condensation reactions and thus the most common methods for the synthesis of 2-(2-oxoindolin-3-ylidene)malononitriles are the condensation of isatins with malononitrile in the presence of a catalyst, such as piperidine acetate (Kayukov et al., 2011), DBU, $\mathrm{Al}_{2} \mathrm{O}_{3}, \mathrm{~N}\left(\mathrm{CH}_{2} \mathrm{CH}_{2} \mathrm{OH}\right)_{3}$ or chitosan (Abdelhamid, 2009). Over the past few years, molecular iodine has emerged as powerful catalyst in various organic transformations (Kidwai et al., 2007). As well as having the advantage of being inexpensive, non-toxic, and nature friendly, iodine affords the desired products in good to excellent yields with high selectivity.<smiles>CCN1C(=O)C(=O)c2ccccc21</smiles><smiles>CCN1C(=O)C(=C([N])[N])c2ccccc21</smiles>

As a continuation of our research on the synthesis, functionilization, physico-chemical and biological properties of indole derivatives (Al Mamari et al., 2012a,b,c,d; Rayni et al., 2017, 2017a,b; Zarrok et al., 2012), we report our results on the 
Knoevenagel condensation of 1-decylindoline-2,3-dione with malononitrile using molecular iodine as catalyst.

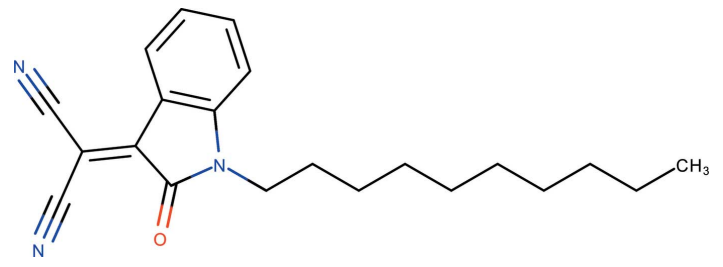

\section{Structural commentary}

The 1-decyl substituent in the title compound (Fig. 1) is fully extended in the crystal and the head end is nearly perpendicular to the plane of the five-membered ring as shown by the $\mathrm{C} 8-\mathrm{N} 1-\mathrm{C} 12-\mathrm{C} 13$ torsion angle of $112.3(2)^{\circ}$. The indole portion is not quite planar, as indicated by the dihedral angle of $1.64(10)^{\circ}$ between the constituent rings and the r.m.s. deviation of $0.015 \AA$. As expected, the propanedinitrile group is essentially coplanar with the five-membered ring, the $\mathrm{C} 8-$ C7-C9-C11 torsion angle being $179.71(17)^{\circ}$.

\section{Supramolecular features}

The molecules pack with the 1-decyl chains intercalating to form large hydrophobic bands (Fig. 2) approximately parallel to the $b$-axis direction. The indole portion participates in offset $\pi-\pi$-stacking interactions in the $b$-axis direction between the five-membered ring in one molecule and the sixmembered ring in the next (Fig. 3) with a centroid-centroid distance of 3.6178 (11) $\AA$ and a dihedral angle of $1.64(10)^{\circ}$. Reinforcing this is a $\mathrm{C}=\mathrm{O} \cdots \pi$ (ring) interaction between $\mathrm{C} 8=\mathrm{O} 1$ and the five-membered ring in the adjacent molecule along the $b$-axis direction (Fig. 3 ) with a C $\cdots$ centroid distance of 3.447 (2) $\AA$.

\section{Database survey}

A search of the Cambridge Structural Database (Version 5.39 with updates through May 2018; Groom et al., 2016) with the fragment shown in Fig. 4 yielded 133 hits of which 34 are close to the title compound in that the substituents on the methyl-

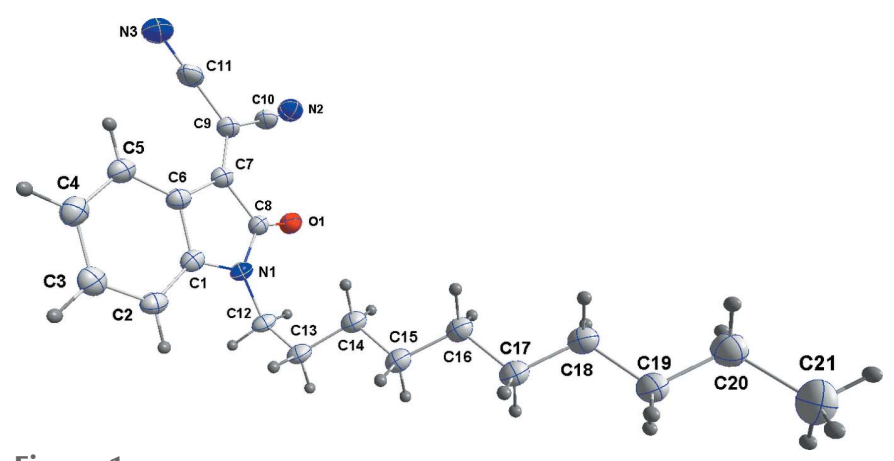

Figure 1

The title molecule with the labelling scheme and $50 \%$ probability ellipsoids.

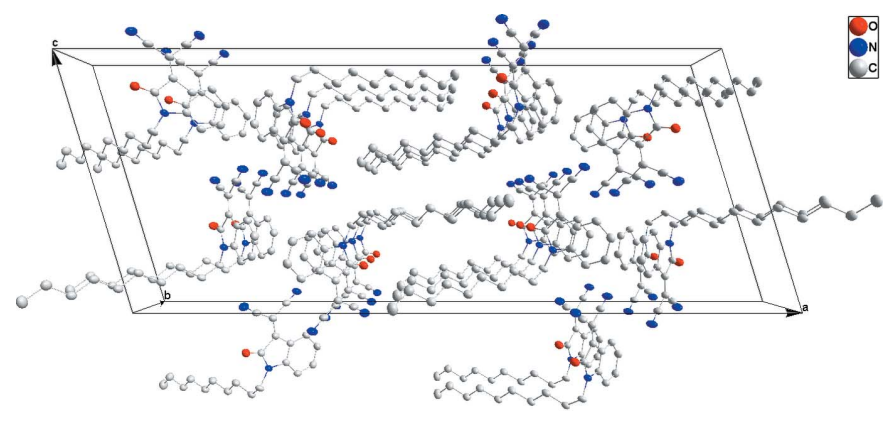

Figure 2

The packing viewed along the $b$ axis.

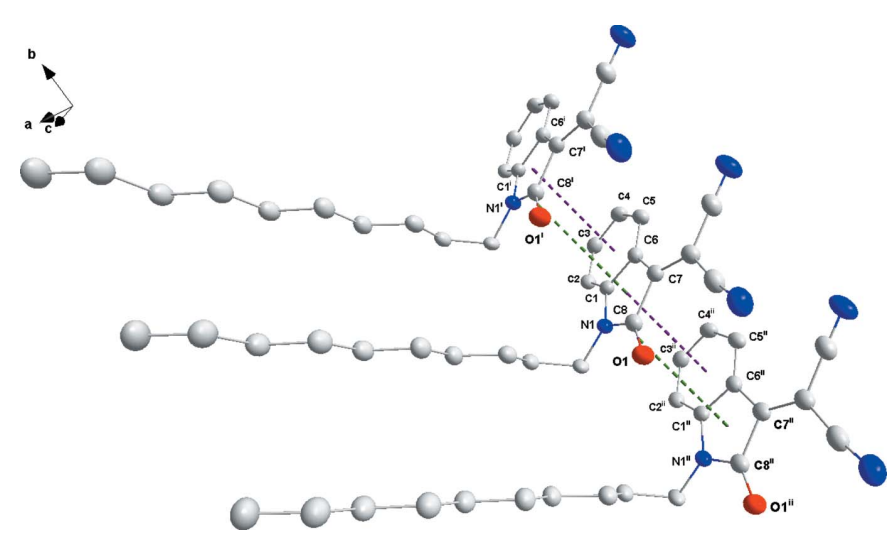

Figure 3

Detail of the offset $\pi$ - $\pi$-stacking (purple dotted lines) and $\mathrm{C}=\mathrm{O} \cdots \pi$ (ring) (green dotted lines) interactions [symmetry codes: (i) $x,-1+y, z$; (ii) $x, 1+y, z]$.

idene carbon are relatively small in size. The closest analogues are $2\left[R=\mathrm{CH}_{3}\right.$ (Wang et al., 2013); $\left(\mathrm{CH}_{2}\right)_{5} \mathrm{CH}_{3}$ (Rayni et al., 2017b)], 3 (Hu et al., 2014) and 4 (Lian et al., 2012) although there are also some interesting related compounds such as $\mathbf{5}[R$ $=\left(\mathrm{CH}_{2}\right)_{5} \mathrm{CH}_{3}$; Hasegawa et al., 2015), $\left(\mathrm{CH}_{2}\right)_{9} \mathrm{CH}_{3}$ (Bogdanov<smiles>C=C1C(=O)N(C)c2ccccc21</smiles>

search fragment<smiles>CCOC(=O)/C(C#N)=C1\C(=O)N(Cc2ccccc2)c2ccccc21</smiles>

Figure 4

Search fragment and related compounds. 
Table 1

The B3LYP-optimized and X-ray structural parameters for $\mathbf{1}\left(\AA{ }^{\circ}{ }^{\circ}\right)$.

\begin{tabular}{lll}
\hline & B3LYP & X-ray \\
\hline C1-C6 & 1.421 & $1.410(2)$ \\
C6-C5 & 1.391 & $1.393(3)$ \\
C5-C4 & 1.404 & $1.389(3)$ \\
C4-C3 & 1.402 & $1.386(3)$ \\
C3-C2 & 1.398 & $1.396(3)$ \\
C2-C1 & 1.402 & $1.377(3)$ \\
C1-N1 & 1.401 & $1.406(2)$ \\
N1-C8 & 1.386 & $1.372(2)$ \\
C8-O1 & 1.220 & $1.214(2)$ \\
C8-C7 & 1.522 & $1.520(2)$ \\
C7-C6 & 1.450 & $1.440(2)$ \\
C7-C9 & 1.396 & $1.350(3)$ \\
C9-C10 & 1.437 & $1.437(3)$ \\
C9-C11 & 1.436 & $1.444(3)$ \\
C10-N2 & 1.165 & $1.147(3)$ \\
C11-N3 & 1.166 & $1.142(3)$ \\
N1-C12 & 1.461 & $1.461(2)$ \\
C12-C13 & $1.536(2)$ \\
C7-C8-N1 & \\
C11-C9-C10 & & $105.90(15)$ \\
C8-N1-C1 & 106.1 & $114.51(16)$ \\
\hline
\end{tabular}

et al., 2014) and $\left(\mathrm{CH}_{2}\right)_{3} \mathrm{CH}_{3}$ (Yuan \& Fang, 2011), $\left(\mathrm{CH}_{2}\right)_{6} \mathrm{Br}$ Bogdanov et al., 2013)]. In these, the indole fragment varies from being planar to having a dihedral angle between the two constituent rings of up to $3.30^{\circ}$. The substituent on the ring nitrogen atom is generally in an extended conformation with the head end nearly perpendicular to the plane of the fivemembered ring with torsion angles corresponding to the $\mathrm{C} 8-$ $\mathrm{N} 1-\mathrm{C} 12-\mathrm{C} 13$ torsion angle in the title compound varying from $73.4-104.8^{\circ}$.

\section{DFT optimization}

The structure in the gas phase of the title compound was optimized by means of density functional theory (DFT). The DFT calculation was performed by the hybrid B3LYP method, which is based on the idea of Becke and considers a mixture of the exact (Hartree-Fock) and DFT exchange utilizing the B3 functional, together with the LYP correlation functional (Becke, 1993; Lee et al., 1988; Miehlich et al., 1989). The B3LYP calculation was performed in conjunction with the basis set DZVP (Godbout et al., 1992). It is noteworthy to mention that the double- $\xi$ basis set used was designed for a DFT calculation. After obtaining the converged geometry, the harmonic vibrational frequencies were calculated at the same theoretical level to confirm that the number of imaginary frequencies is zero for the stationary point. Both the geometry optimization and harmonic vibrational frequency analysis of the title compound were performed with the Gaussian16 program (Frisch et al., 2016).

The result of the B3LYP geometry optimization for the title compound was compared with that determined in the crystallographic study. The B3LYP-optimized geometry of the title compound is shown in Fig. 5 with selected geometric parameters of the gas-phase and the solid-phase structures summarized in Table 1. These show that the gas-phase struc-

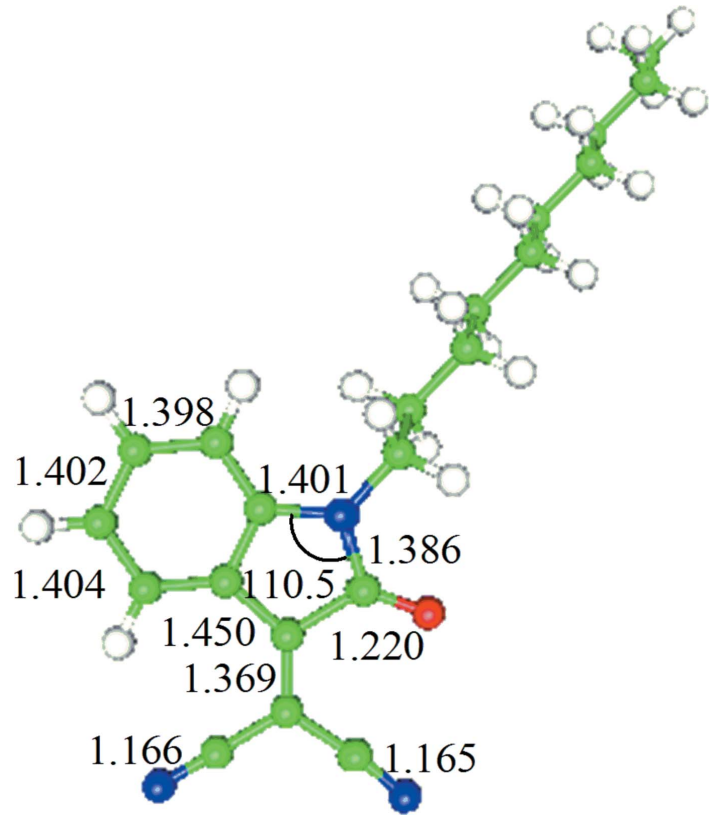

Figure 5

The B3LYP-optimized geometry of the title compound $\left(\AA{ }^{\circ}\right)$.

ture shows a small deviation from the solid-phase one (Reichman et al., 1969; Liao \& Zhang, 1998).

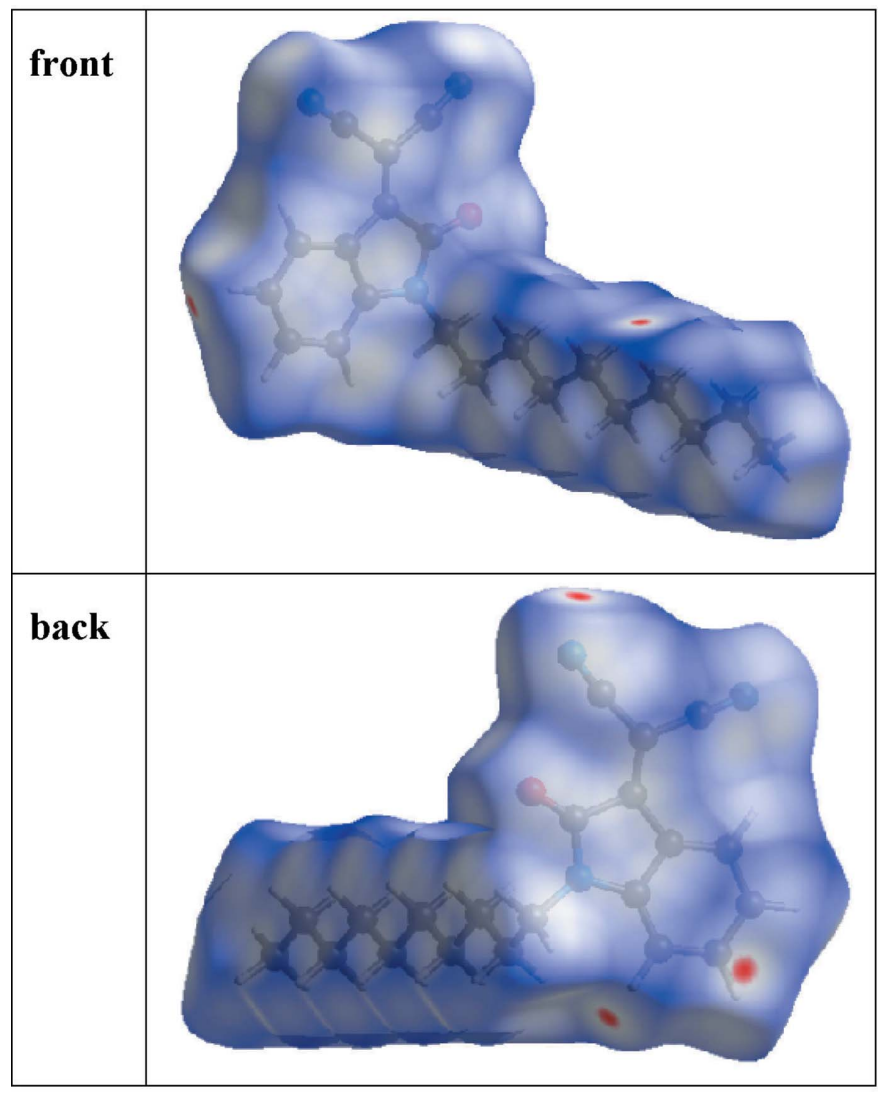

Figure 6

The $d_{\text {norm }}$ Hirshfeld surface of the title compound (red: negative, white: zero, blue: positive; scale: -0.0774 to 1.3395 a.u.). 


\section{Hirshfeld surface calculations}

Both the definition of a molecule in a condensed phase and the recognition of distinct entities in molecular liquids and crystals are fundamental concepts in chemistry. Based on Hirshfeld's partitioning scheme, a method was proposed to divide the electron distribution in a crystalline phase into molecular fragments (Spackman \& Byrom, 1997; McKinnon et al., 2004; Spackman \& Jayatilaka, 2009). This method partitioned the crystal into regions where the electron distribution of a sum of spherical atoms for the molecule dominates over the corresponding sum of the crystal. As it is derived from Hirshfeld's stockholder partitioning, the molecular surface is named as the Hirshfeld surface. In this study, the Hirshfeld surface analysis of the title compound was performed utilizing the CrystalExplorer program (Turner et al., 2017).

The standard resolution molecular Hirshfeld surface $\left(d_{\text {norm }}\right)$ of the title compound is shown in Fig. 6 . The 3D $d_{\text {norm }}$ surface is used to identify close intermolecular interactions. The value of $d_{\text {norm }}$ is negative (positive) when intermolecular contacts are shorter (longer) than the van der Waals radii. The $d_{\text {norm }}$ value is mapped onto the Hirshfeld surface using red, white and blue. The red regions represent closer contacts with a negative $d_{\text {norm }}$ value while the blue regions represent longer contacts with a positive $d_{\text {norm }}$ value and the white regions represent contacts equal to the van der Waals separation and have a $d_{\text {norm }}$ value of zero. As shown in Fig. 6, the major interactions in the title compound are intermolecular $\mathrm{H} \cdots \mathrm{O}$ and $\mathrm{H} \cdots \mathrm{N}$ hydrogen bonds.

The 2D fingerprint plots highlight particular atom-pair contacts and enable the separation of contributions from different interaction types that overlap in the full fingerprint.

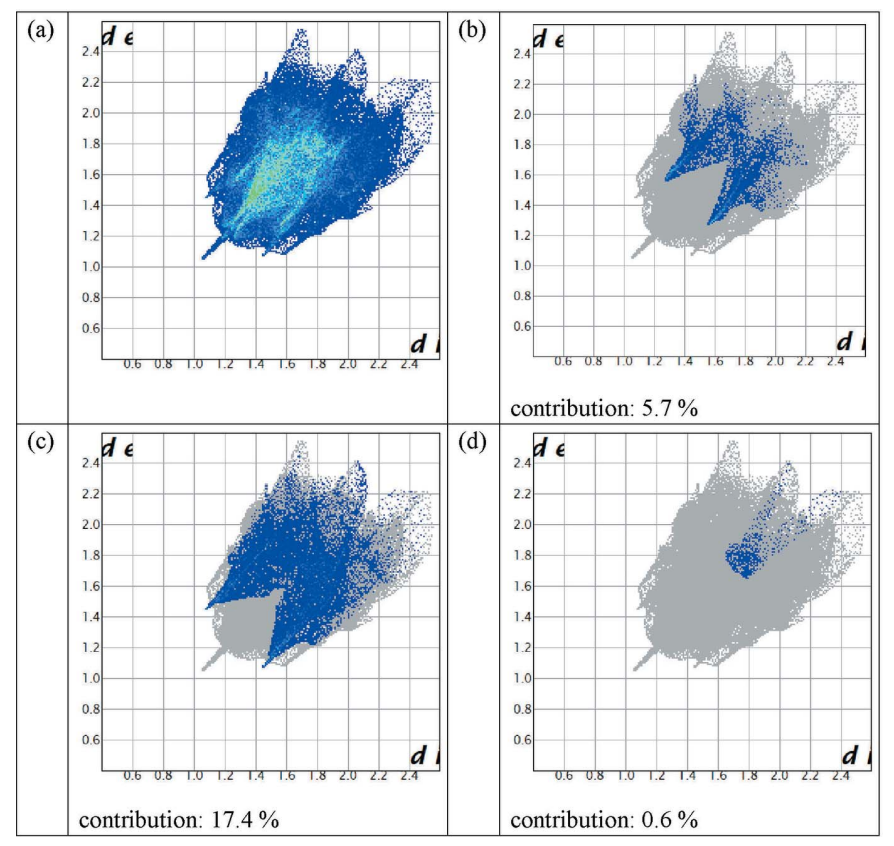

Figure 7

The two-dimensional fingerprint plot of the title compound (a) full and decomposed into $(b) \mathrm{H} \cdots \mathrm{O} / \mathrm{O} \cdots \mathrm{H}$ contacts, $(c) \mathrm{H} \cdots \mathrm{N} / \mathrm{N} \cdots \mathrm{H}$ contacts and $(d) \mathrm{N} \cdots \mathrm{N}$ contacts.
Table 2

Experimental details.

\begin{tabular}{|c|c|}
\hline \multicolumn{2}{|l|}{ Crystal data } \\
\hline Chemical formula & $\mathrm{C}_{21} \mathrm{H}_{25} \mathrm{~N}_{3} \mathrm{O}$ \\
\hline$M_{\mathrm{r}}$ & 335.44 \\
\hline Crystal system, space group & Monoclinic, $C 2 / c$ \\
\hline Temperature (K) & 150 \\
\hline$a, b, c(\AA)$ & $\begin{array}{l}44.4837(12), 4.7293(1), \\
\quad 18.3432(5)\end{array}$ \\
\hline$\beta\left(^{\circ}\right)$ & $106.965(2)$ \\
\hline$V\left(\AA^{3}\right)$ & 3691.05 (17) \\
\hline$Z$ & 8 \\
\hline Radiation type & $\mathrm{Cu} K \alpha$ \\
\hline$\mu\left(\mathrm{mm}^{-1}\right)$ & 0.59 \\
\hline Crystal size $(\mathrm{mm})$ & $0.29 \times 0.08 \times 0.03$ \\
\hline \multicolumn{2}{|l|}{ Data collection } \\
\hline Diffractometer & $\begin{array}{l}\text { Bruker D8 VENTURE PHOTON } \\
100 \text { CMOS }\end{array}$ \\
\hline Absorption correction & $\begin{array}{l}\text { Multi-scan (TWINABS; Sheldrick, } \\
\text { 2009) }\end{array}$ \\
\hline$T_{\min }, T_{\max }$ & $0.75,0.98$ \\
\hline $\begin{array}{l}\text { No. of measured, independent and } \\
\text { observed }[I>2 \sigma(I)] \text { reflections }\end{array}$ & $25556,3592,2656$ \\
\hline$R_{\text {int }}$ & 0.054 \\
\hline$(\sin \theta / \lambda)_{\max }\left(\AA^{-1}\right)$ & 0.618 \\
\hline \multicolumn{2}{|l|}{ Refinement } \\
\hline$R\left[F^{2}>2 \sigma\left(F^{2}\right)\right], w R\left(F^{2}\right), S$ & $0.051,0.134,1.05$ \\
\hline No. of reflections & 3592 \\
\hline No. of parameters & 326 \\
\hline $\mathrm{H}$-atom treatment & All $\mathrm{H}$-atom parameters refined \\
\hline$\Delta \rho_{\max }, \Delta \rho_{\min }\left(\mathrm{e} \AA^{-3}\right)$ & $0.24,-0.20$ \\
\hline
\end{tabular}

Computer programs: APEX3 and SAINT (Bruker, 2016), SHELXT (Sheldrick, 2015a), SHELXL 2014/7 (Sheldrick, 2015b), DIAMOND (Brandenburg \& Putz, 2012) and SHELXTL (Sheldrick, 2008).

Using the standard 0.6-2.6 view with the $d_{\mathrm{e}}$ and $d_{\mathrm{i}}$ distance scales displayed on the graph axes and including the reciprocal contacts, the contribution of the $\mathrm{H} \cdots \mathrm{N}$ contacts is larger than that of the $\mathrm{H} \cdots \mathrm{O}$ contacts (Fig. 7). Interestingly, we found that there is a negligible contribution of $\mathrm{N} \cdots \mathrm{N}$ contacts (Govers, 1975; Cartwright \& Wilkinson, 2010). This interaction might be considered as a stabilizing hyperconjugative one between a $\pi$-bonding orbital of one $\mathrm{C} \equiv \mathrm{N}$ group and a $\pi^{*}$-bonding orbital of another $\left[\mathrm{C} \equiv \mathrm{N}\right.$ group $\pi(\mathrm{CN}) \rightarrow \pi^{*}\left(\mathrm{C}^{\prime} \mathrm{N}^{\prime}\right)$; Jeong \& Kwon, 2000].

\section{Synthesis and crystallization}

A mixture of 1-decylindole-2,3-dione $(0,5 \mathrm{~g}, 2.1 \mathrm{mmol})$, malononitrile $(0,14 \mathrm{~g}, 2.1 \mathrm{mmol})$, and $\mathrm{I}_{2}(0.05 \mathrm{~g}, 0.21 \mathrm{mmol})$ in ethanol $(10 \mathrm{~mL})$ was heated at $333 \mathrm{~K}$. After completion of the reaction (monitored by TLC), the mixture was treated with aqueous $\mathrm{Na}_{2} \mathrm{~S}_{2} \mathrm{O}_{3}$ solution and extracted with ethyl acetate ( 2 $\times 10 \mathrm{~mL}$ ). The extract was dried over sodium sulfate, filtered and the solvent was evaporated in vacuo. The purified product was recrystallized from ethanol solution to afford the title compound as orange, plate-like crystals.

\section{Refinement}

Crystal data, data collection and structure refinement details are summarized in Table 2. Trial refinements of the model with 
the one-component reflection file extracted from the full twinned data with TWINABS and with the full, two-component reflection file indicated that the former gave better results both in terms of lower values of $R_{1}$ and $w R_{2}$ and in lower s.u. values for derived parameters.

\section{Funding information}

The support of NSF-MRI grant No. 1228232 for the purchase of the diffractometer and Tulane University for support of the Tulane Crystallography Laboratory are gratefully acknowledged. The publication was prepared with the support of the RUDN University Program 5-100.

\section{References}

Abdelhamid, I. A. (2009). Synlett, pp. 625-627.

Al Mamari, K., Ennajih, H., Bouhfid, R., Essassi, E. M. \& Ng, S. W. (2012b). Acta Cryst. E68, o1664.

Al Mamari, K., Ennajih, H., Bouhfid, R., Essassi, E. M. \& Ng, S. W. (2012c). Acta Cryst. E68, o1638.

Al Mamari, K., Ennajih, H., Bouhfid, R., Essassi, E. M. \& Ng, S. W. (2012d). Acta Cryst. E68, o1637.

Al Mamari, K., Ennajih, H., Zouihri, H., Bouhfid, R., Ng, S. W. \& Essassi, E. M. (2012a). Tetrahedron Lett. 53, 2328-2331.

Becke, A. D. (1993). J. Chem. Phys. 98, 5648-5652.

Bogdanov, A. V., Pashirova, T. N., Musin, L. I., Krivolapov, D. B., Zakharova, L. Ya., Mironov, V. F. \& Konovalov, A. I. (2014). Chem. Phys. Lett. 594, 69-73.

Bogdanov, A. V., Yusupova, G. G., Romanova, I. P., Latypov, S. K., Krivolapov, D. P., Mironov, V. F. \& Sinyashin, O. G. (2013). Synthesis, 45, 668-672.

Brandenburg, K. \& Putz, H. (2012). DIAMOND, Crystal Impact GbR, Bonn, Germany.

Bruker (2016). APEX3, SAINT and SADABS. Bruker AXS, Inc., Madison, Wisconsin, USA.

Cartwright, M. \& Wilkinson, J. (2010). Propellants, Explosives, Pyrotech. 35, 326-332.

Frisch, M. J., Trucks, G. W., Schlegel, H. B., Scuseria, G. E., Robb, M. A., Cheeseman, J. R., Scalmani, G., Barone, V., Petersson, G. A., Nakatsuji, H., Li, X., Caricato, M., Marenich, A. V., Bloino, J., Janesko, B. G., Gomperts, R., Mennucci, B., Hratchian, H. P., Ortiz, J. V., Izmaylov, A. F., Sonnenberg, J. L., Williams-Young, D., Ding, F., Lipparini, F., Egidi, F., Goings, J., Peng, B., Petrone, A., Henderson, T., Ranasinghe, D., Zakrzewski, V. G., Gao, J., Rega, N., Zheng, G., Liang, W., Hada, M., Ehara, M., Toyota, K., Fukuda, R., Hasegawa, J., Ishida, M., Nakajima, T., Honda, Y., Kitao, O., Nakai, H., Vreven, T., Throssell, K., Montgomery, J. A., Peralta, Jr., J. E., Ogliaro, F., Bearpark, M. J., Heyd, J. J., Brothers, E. N., Kudin, K. N., Staroverov, V. N., Keith, T. A., Kobayashi, R., Normand, J., Raghavachari, K., Rendell, A. P., Burant, J. C., Iyengar, S. S., Tomasi, J., Cossi, M., Millam, J. M., Klene, M., Adamo, C., Cammi, R., Ochterski, J. W., Martin, R. L., Morokuma, K., Farkas,
O., Foresman, J. B. \& Fox, D. J. (2016). Gaussian 16, Revision A.03. Gaussian, Inc., Wallingford CT.

Godbout, N., Salahub, N. R., Andzelm, J. \& Wimmer, E. (1992). Can. J. Chem. 70, 560-571.

Govers, H. A. J. (1975). Acta Cryst. A31, 380-385.

Groom, C. R., Bruno, I. J., Lightfoot, M. P. \& Ward, S. C. (2016). Acta Cryst. B72, 171-179.

Hasegawa, T., Ashizawa, M. \& Matsumoto, H. (2015). RSC Adv. 5, 61035-61043.

Hu, F.-L., Wei, Y. \& Shi, M. (2014). Chem. Commun. 50, 8912-8914. Jeong, M. \& Kwon, Y. (2000). Chem. Phys. Lett. 324, 183-188.

Jones, G. (1967). Organic Reactions, Vol. 15, pp. 204-599. New York: Wiley.

Kayukov, Y. S., Kayukova, O. V., Kalyagina, E. S., Bardasov, I. N., Ershov, O. V., Nasakin, O. E. \& Tafeenko, V. A. (2011). Russ. J. Org. Chem. 47, 392-401.

Kidwai, M., Mothsra, P., Bansal, V., Somvanshi, R. K., Ethayathulla, A. S., Dey, S. \& Singh, T. P. (2007). J. Mol. Catal. A Chem. 265, 177182.

Lee, C., Yang, W. \& Parr, R. G. (1988). Phys. Rev. B, 37, 785-789.

Lian, Z., Wei, Y. \& Shi, M. (2012). Tetrahedron, 68, 2401-2408.

Liao, M.-S. \& Zhang, Q.-E. (1998). J. Phys. Chem. A, 102, 1064710654.

McKinnon, J. J., Spackman, M. A. \& Mitchell, A. S. (2004). Acta Cryst. B60, 627-668.

Miehlich, B., Savin, A., Stoll, H. \& Preuss, H. (1989). Chem. Phys. Lett. 157, 200-206.

Pandeya, S. N., Smitha, S., Jyoti, M. \& Sridhar, S. K. (2005). Acta Pharm. 55, 27-46.

Rayni, I., El Bakri, Y., Essassi, E. M. \& Mague, J. T. (2017). J. Mar. Chim. Heterocycl. 16, 207-214.

Rayni, I., El Bakri, Y., Sebhaoui, J., El Bourakadi, K., Essassi, E. M. \& Mague, J. T. (2017a). IUCrData, 2, x170315.

Rayni, I., El Bakri, Y., Sebhaoui, J., El Bourakadi, K., Essassi, E. M. \& Mague, J. T. (2017b). IUCrData, 2, x170706.

Reichman, S. \& Schreiner, F. (1969). J. Chem. Phys. 51, 2355-2358.

Sheldrick, G. M. (2008). Acta Cryst. A64, 112-122.

Sheldrick, G. M. (2009). TWINABS. University of Göttingen, Göttingen, Germany.

Sheldrick, G. M. (2015a). Acta Cryst. A71, 3-8.

Sheldrick, G. M. (2015b). Acta Cryst. C71, 3-8.

Spackman, M. A. \& Byrom, P. G. (1997). Chem. Phys. Lett. 267, 215220.

Spackman, M. A. \& Jayatilaka, D. (2009). CrystEngComm, 11, 1932.

Turner, M. J., McKinnon, J. J., Wolff, S. K., Grimwood, D. J., Spackman, P. R., Jayatilaka, D. \& Spackman, M. A. (2017). CrystalExplorer17. University of Western Australia.

Wang, D.-C., Tang, W., Su, P. \& Ou-Yang, P.-K. (2013). Acta Cryst. E69, o1095.

Yuan, M.-S. \& Fang, Q. (2011). Acta Cryst. E67, o52.

Zarrok, H., Al Mamari, K., Zarrouk, A., Salghi, R., Hammouti, B., Al-Deyab, S. S., Essassi, E. M., Bentiss, F. \& Oudda, H. (2012). Int. J. Electrochem. Sci. 7, 10338-10357. 


\section{supporting information}

Acta Cryst. (2019). E75, 21-25 [https://doi.org/10.1107/S2056989018017267]

Synthesis, crystal structure, DFT calculations and Hirshfeld surface analysis of

2-(1-decyl-2-oxoindolin-3-ylidene)propanedinitrile

Ibtissam Rayni, Youness El Bakri, Chin-Hung Lai, L'houssaine El Ghayati, El Mokhtar Essassi and

Joel T. Mague

Computing details

Data collection: APEX3 (Bruker, 2016); cell refinement: SAINT (Bruker, 2016); data reduction: SAINT (Bruker, 2016); program(s) used to solve structure: SHELXT (Sheldrick, 2015a); program(s) used to refine structure: SHELXL 2014/7 (Sheldrick, 2015b); molecular graphics: DIAMOND (Brandenburg \& Putz, 2012); software used to prepare material for publication: SHELXTL (Sheldrick, 2008).

2-(1-decyl-2-oxoindolin-3-ylidene)propanedinitrile

Crystal data

$\mathrm{C}_{21} \mathrm{H}_{25} \mathrm{~N}_{3} \mathrm{O}$

$M_{r}=335.44$

Monoclinic, $C 2 / c$

$a=44.4837$ (12) $\AA$

$b=4.7293(1) \AA$

$c=18.3432(5) \AA$

$\beta=106.965(2)^{\circ}$

$V=3691.05(17) \AA^{3}$

$Z=8$

$F(000)=1440$

$D_{\mathrm{x}}=1.207 \mathrm{Mg} \mathrm{m}^{-3}$

$\mathrm{Cu} K \alpha$ radiation, $\lambda=1.54178 \AA$

Cell parameters from 9886 reflections

$\theta=4.2-72.3^{\circ}$

$\mu=0.59 \mathrm{~mm}^{-1}$

$T=150 \mathrm{~K}$

Plate, orange

$0.29 \times 0.08 \times 0.03 \mathrm{~mm}$

\section{Data collection}

Bruker D8 VENTURE PHOTON 100 CMOS diffractometer

Radiation source: INCOATEC I $\mu \mathrm{S}$ micro-focus source

Mirror monochromator

Detector resolution: 10.4167 pixels $\mathrm{mm}^{-1}$

$\omega$ scans

Absorption correction: multi-scan

(TWINABS; Sheldrick, 2009)

$T_{\min }=0.75, T_{\max }=0.98$

25556 measured reflections

3592 independent reflections

2656 reflections with $I>2 \sigma(I)$

$R_{\text {int }}=0.054$

$\theta_{\max }=72.3^{\circ}, \theta_{\min }=2.1^{\circ}$

$h=-54 \rightarrow 5$

$k=-5 \rightarrow 5$

$l=-21 \rightarrow 22$

Primary atom site location: structure-invariant direct methods

Secondary atom site location: difference Fourier map

Hydrogen site location: difference Fourier map

All $\mathrm{H}$-atom parameters refined

$w=1 /\left[\sigma^{2}\left(F_{\mathrm{o}}^{2}\right)+(0.0647 P)^{2}+0.6236 P\right]$

where $P=\left(F_{\mathrm{o}}{ }^{2}+2 F_{\mathrm{c}}{ }^{2}\right) / 3$ 
$(\Delta / \sigma)_{\max }=0.001$

$\Delta \rho_{\min }=-0.20$ e $\AA^{-3}$

$\Delta \rho_{\max }=0.24$ e $\AA^{-3}$

Special details

Experimental. Analysis of 1401 reflections having $\mathrm{I} / \sigma(\mathrm{I})>15$ and chosen from the full data set with $C E L L \_N O W$ (Sheldrick, 2008) showed the crystal to belong to the triclinic system and to be twinned by a $180^{\circ}$ rotation about the $a$ axis. The raw data were processed using the multi-component version ofSAINT under control of the two-component orientation file generated by $C E L L+N O W$.

Geometry. All esds (except the esd in the dihedral angle between two 1.s. planes) are estimated using the full covariance matrix. The cell esds are taken into account individually in the estimation of esds in distances, angles and torsion angles; correlations between esds in cell parameters are only used when they are defined by crystal symmetry. An approximate (isotropic) treatment of cell esds is used for estimating esds involving l.s. planes.

Refinement. Refinement of $\mathrm{F}^{2}$ against ALL reflections. The weighted R-factor $\mathrm{wR}$ and goodness of fit $\mathrm{S}$ are based on $\mathrm{F}^{2}$, conventional $\mathrm{R}$-factors $\mathrm{R}$ are based on $\mathrm{F}$, with $\mathrm{F}$ set to zero for negative $\mathrm{F}^{2}$. The threshold expression of $\mathrm{F}^{2}>2 \operatorname{sigma}\left(\mathrm{F}^{2}\right)$ is used only for calculating R-factors(gt) etc. and is not relevant to the choice of reflections for refinement. R-factors based on $\mathrm{F}^{2}$ are statistically about twice as large as those based on F, and R- factors based on ALL data will be even larger. Trial refinements with the single-component reflection file extracted from the full data set with TWINABS and with the complete two-component reflection file showed the former refinement to be the better one.

Fractional atomic coordinates and isotropic or equivalent isotropic displacement parameters $\left(\hat{A}^{2}\right)$

\begin{tabular}{|c|c|c|c|c|}
\hline & $x$ & $y$ & $z$ & $U_{\text {iso }} * / U_{\text {eq }}$ \\
\hline O1 & $0.36446(3)$ & -0.1282 & $0.67635(8)$ & $0.0362(3)$ \\
\hline N1 & 0.34709 & $0.1596(3)$ & $0.75794(8)$ & $0.0271(3)$ \\
\hline $\mathrm{N} 2$ & $0.34275(4)$ & $-0.2025(5)$ & $0.49015(10)$ & $0.0470(5)$ \\
\hline N3 & $0.27212(4)$ & $0.4371(5)$ & $0.45464(10)$ & $0.0495(5)$ \\
\hline $\mathrm{C} 1$ & $0.32328(4)$ & $0.3634(4)$ & $0.74915(10)$ & $0.0262(4)$ \\
\hline $\mathrm{C} 2$ & $0.31433(4)$ & $0.5037(4)$ & $0.80525(10)$ & $0.0286(4)$ \\
\hline $\mathrm{H} 2$ & $0.3249(5)$ & $0.469(5)$ & $0.8594(13)$ & $0.032(5)^{*}$ \\
\hline $\mathrm{C} 3$ & $0.28988(4)$ & 0.6993 (4) & $0.78192(11)$ & $0.0317(4)$ \\
\hline $\mathrm{H} 3$ & $0.2834(5)$ & $0.807(5)$ & 0.8209 (13) & $0.041(6)^{*}$ \\
\hline $\mathrm{C} 4$ & $0.27473(4)$ & $0.7480(4)$ & $0.70550(11)$ & $0.0319(4)$ \\
\hline $\mathrm{H} 4$ & $0.2582(5)$ & $0.880(5)$ & $0.6893(12)$ & $0.034(5)^{*}$ \\
\hline $\mathrm{C} 5$ & $0.28324(4)$ & $0.5999(4)$ & $0.64921(11)$ & $0.0303(4)$ \\
\hline H5 & $0.2723(5)$ & $0.633(5)$ & $0.5964(14)$ & $0.039(6)^{*}$ \\
\hline C6 & $0.30782(4)$ & $0.4059(4)$ & $0.67108(10)$ & $0.0267(4)$ \\
\hline $\mathrm{C} 7$ & $0.32211(4)$ & $0.2166(4)$ & $0.62943(10)$ & $0.0273(4)$ \\
\hline $\mathrm{C} 8$ & $0.34757(4)$ & 0.0565 & $0.68839(10)$ & $0.0283(4)$ \\
\hline C9 & $0.31586(4)$ & 0.1659 (4) & $0.55402(10)$ & $0.0301(4)$ \\
\hline $\mathrm{C} 10$ & $0.33204(4)$ & $-0.0402(5)$ & $0.52187(10)$ & $0.0346(4)$ \\
\hline $\mathrm{C} 11$ & $0.29150(4)$ & $0.3188(5)$ & $0.49872(11)$ & $0.0354(4)$ \\
\hline $\mathrm{C} 12$ & $0.36711(4)$ & $0.0513(4)$ & $0.83047(10)$ & $0.0296(4)$ \\
\hline $\mathrm{H} 12 \mathrm{~A}$ & $0.3786(5)$ & $-0.129(5)$ & $0.8172(12)$ & $0.036(6)^{*}$ \\
\hline H12B & $0.3527(5)$ & $0.000(5)$ & $0.8620(12)$ & $0.032(5)^{*}$ \\
\hline $\mathrm{C} 13$ & $0.39191(4)$ & $0.2618(4)$ & $0.87386(10)$ & $0.0298(4)$ \\
\hline $\mathrm{H} 13 \mathrm{~A}$ & $0.4035(5)$ & $0.161(5)$ & $0.9230(13)$ & $0.039(6)^{*}$ \\
\hline H13B & $0.3805(5)$ & $0.432(5)$ & $0.8878(12)$ & $0.032(5)^{*}$ \\
\hline $\mathrm{C} 14$ & $0.41561(4)$ & $0.3492(4)$ & $0.83250(11)$ & $0.0317(4)$ \\
\hline H14A & $0.4257(5)$ & $0.170(5)$ & 0.8154 (13) & $0.044(6)^{*}$ \\
\hline H14B & $0.4049(5)$ & $0.453(5)$ & $0.7845(13)$ & $0.034(5)^{*}$ \\
\hline
\end{tabular}




$\begin{array}{lllll}\text { C15 } & 0.44154(4) & 0.5345(4) & 0.88269(11) & 0.0323(4) \\ \text { H15A } & 0.4527(5) & 0.429(5) & 0.9310(14) & 0.044(6)^{*} \\ \text { H15B } & 0.4321(5) & 0.709(5) & 0.9023(12) & 0.034(5)^{*} \\ \text { C16 } & 0.46614(4) & 0.6289(4) & 0.84511(11) & 0.0335(4) \\ \text { H16A } & 0.4555(6) & 0.739(5) & 0.7968(14) & 0.047(6)^{*} \\ \text { H16B } & 0.4759(5) & 0.461(5) & 0.8270(12) & 0.040(6)^{*} \\ \text { C17 } & 0.49228(4) & 0.8068(5) & 0.89736(11) & 0.0330(4) \\ \text { H17A } & 0.5025(6) & 0.692(5) & 0.9449(15) & 0.052(7)^{*} \\ \text { H17B } & 0.4824(5) & 0.979(5) & 0.9136(13) & 0.042(6)^{*} \\ \text { C18 } & 0.51688(4) & 0.9018(5) & 0.85996(11) & 0.0355(5) \\ \text { H18A } & 0.5257(6) & 0.730(5) & 0.8419(14) & 0.048(6)^{*} \\ \text { H18B } & 0.5063(6) & 1.017(5) & 0.8130(15) & 0.051(7)^{*} \\ \text { C19 } & 0.54344(4) & 1.0786(5) & 0.91077(11) & 0.0341(4) \\ \text { H19A } & 0.5547(5) & 0.968(5) & 0.9594(14) & 0.046(6)^{*} \\ \text { H19B } & 0.5334(5) & 1.249(5) & 0.9312(13) & 0.041(6)^{*} \\ \text { C20 } & 0.56740(5) & 1.1722(5) & 0.87134(13) & 0.0403(5) \\ \text { H20A } & 0.5768(6) & 0.999(6) & 0.8527(14) & 0.052(7)^{*} \\ \text { H20B } & 0.5552(5) & 1.280(5) & 0.8244(14) & 0.046(6)^{*} \\ \text { C21 } & 0.59341(5) & 1.3554(6) & 0.92162(16) & 0.0483(6) \\ \text { H21A } & 0.5840(6) & 1.529(6) & 0.9393(15) & 0.061(8)^{*} \\ \text { H21B } & 0.6081(7) & 1.418(6) & 0.8951(17) & 0.067(8)^{*} \\ \text { H21C } & 0.6055(6) & 1.242(6) & 0.9663(17) & 0.062(8)^{*}\end{array}$

Atomic displacement parameters $\left(\AA^{2}\right)$

\begin{tabular}{lllllll}
\hline & $U^{11}$ & $U^{22}$ & $U^{33}$ & $U^{12}$ & $U^{13}$ & $U^{23}$ \\
\hline $\mathrm{O} 1$ & $0.0383(7)$ & $0.0343(8)$ & $0.0341(7)$ & $0.0090(6)$ & $0.0073(5)$ & $-0.0011(6)$ \\
$\mathrm{N} 1$ & $0.0288(7)$ & $0.0250(8)$ & $0.0238(8)$ & $0.0017(6)$ & $0.0020(5)$ & $0.0023(6)$ \\
$\mathrm{N} 2$ & $0.0512(10)$ & $0.0557(13)$ & $0.0322(9)$ & $0.0028(9)$ & $0.0092(8)$ & $-0.0079(8)$ \\
$\mathrm{N} 3$ & $0.0471(9)$ & $0.0669(14)$ & $0.0292(9)$ & $0.0081(10)$ & $0.0030(7)$ & $0.0083(9)$ \\
$\mathrm{C} 1$ & $0.0261(7)$ & $0.0224(9)$ & $0.0276(9)$ & $-0.0019(7)$ & $0.0039(6)$ & $0.0026(7)$ \\
$\mathrm{C} 2$ & $0.0316(8)$ & $0.0280(10)$ & $0.0245(9)$ & $-0.0024(8)$ & $0.0057(7)$ & $0.0030(7)$ \\
$\mathrm{C} 3$ & $0.0320(8)$ & $0.0307(10)$ & $0.0333(10)$ & $-0.0019(8)$ & $0.0112(7)$ & $-0.0015(8)$ \\
$\mathrm{C} 4$ & $0.0298(8)$ & $0.0293(10)$ & $0.0354(10)$ & $0.0036(8)$ & $0.0074(7)$ & $0.0038(7)$ \\
C5 & $0.0289(8)$ & $0.0309(11)$ & $0.0278(10)$ & $-0.0009(7)$ & $0.0031(7)$ & $0.0048(7)$ \\
C6 & $0.0284(8)$ & $0.0250(9)$ & $0.0247(9)$ & $-0.0028(7)$ & $0.0046(6)$ & $0.0009(7)$ \\
C7 & $0.0282(8)$ & $0.0257(9)$ & $0.0261(9)$ & $-0.0028(7)$ & $0.0049(7)$ & $0.0018(7)$ \\
C8 & $0.0263(8)$ & $0.0293(10)$ & $0.0275(9)$ & $-0.0007(7)$ & $0.0048(6)$ & $0.0011(7)$ \\
C9 & $0.0312(8)$ & $0.0310(10)$ & $0.0260(9)$ & $-0.0016(8)$ & $0.0053(7)$ & $0.0011(7)$ \\
C10 & $0.0363(9)$ & $0.0413(12)$ & $0.0239(9)$ & $-0.0014(9)$ & $0.0050(7)$ & $-0.0023(8)$ \\
C11 & $0.0372(9)$ & $0.0448(12)$ & $0.0228(9)$ & $-0.0028(9)$ & $0.0068(7)$ & $-0.0015(8)$ \\
C12 & $0.0331(8)$ & $0.0265(10)$ & $0.0236(9)$ & $0.0008(8)$ & $-0.0009(7)$ & $0.0061(7)$ \\
C13 & $0.0306(8)$ & $0.0300(10)$ & $0.0240(9)$ & $0.0026(8)$ & $0.0004(7)$ & $0.0012(7)$ \\
C14 & $0.0331(9)$ & $0.0307(10)$ & $0.0271(10)$ & $0.0029(8)$ & $0.0024(7)$ & $-0.0004(8)$ \\
C15 & $0.0314(8)$ & $0.0331(11)$ & $0.0285(10)$ & $0.0005(8)$ & $0.0025(7)$ & $-0.0026(8)$ \\
C16 & $0.0334(9)$ & $0.0348(11)$ & $0.0296(10)$ & $0.0004(8)$ & $0.0046(7)$ & $-0.0037(8)$ \\
C17 & $0.0336(9)$ & $0.0337(11)$ & $0.0283(10)$ & $0.0007(8)$ & $0.0037(7)$ & $-0.0009(8)$ \\
C18 & $0.0368(9)$ & $0.0378(12)$ & $0.0305(10)$ & $0.0005(9)$ & $0.0074(8)$ & $-0.0024(8)$ \\
& & & & & &
\end{tabular}




\begin{tabular}{lllllll}
$\mathrm{C} 19$ & $0.0342(9)$ & $0.0357(12)$ & $0.0306(10)$ & $-0.0003(8)$ & $0.0064(7)$ & $-0.0008(8)$ \\
$\mathrm{C} 20$ & $0.0403(10)$ & $0.0395(13)$ & $0.0417(12)$ & $-0.0015(10)$ & $0.0130(9)$ & $-0.0025(9)$ \\
$\mathrm{C} 21$ & $0.0388(11)$ & $0.0439(14)$ & $0.0613(16)$ & $-0.0052(10)$ & $0.0133(10)$ & $-0.0039(12)$ \\
\hline
\end{tabular}

Geometric parameters $\left(\AA,{ }^{\circ}\right)$

\begin{tabular}{|c|c|c|c|}
\hline $\mathrm{O} 1-\mathrm{C} 8$ & $1.214(2)$ & $\mathrm{C} 13-\mathrm{H} 13 \mathrm{~B}$ & $1.02(2)$ \\
\hline $\mathrm{N} 1-\mathrm{C} 8$ & $1.372(2)$ & $\mathrm{C} 14-\mathrm{C} 15$ & $1.525(3)$ \\
\hline $\mathrm{N} 1-\mathrm{C} 1$ & $1.406(2)$ & $\mathrm{C} 14-\mathrm{H} 14 \mathrm{~A}$ & $1.05(2)$ \\
\hline $\mathrm{N} 1-\mathrm{C} 12$ & $1.461(2)$ & C14-H14B & $1.00(2)$ \\
\hline $\mathrm{N} 2-\mathrm{C} 10$ & $1.147(3)$ & $\mathrm{C} 15-\mathrm{C} 16$ & $1.521(3)$ \\
\hline $\mathrm{N} 3-\mathrm{C} 11$ & $1.142(3)$ & $\mathrm{C} 15-\mathrm{H} 15 \mathrm{~A}$ & $1.01(2)$ \\
\hline $\mathrm{C} 1-\mathrm{C} 2$ & $1.377(3)$ & $\mathrm{C} 15-\mathrm{H} 15 \mathrm{~B}$ & $1.04(2)$ \\
\hline $\mathrm{C} 1-\mathrm{C} 6$ & $1.410(2)$ & $\mathrm{C} 16-\mathrm{C} 17$ & $1.526(3)$ \\
\hline $\mathrm{C} 2-\mathrm{C} 3$ & $1.396(3)$ & $\mathrm{C} 16-\mathrm{H} 16 \mathrm{~A}$ & $1.02(3)$ \\
\hline $\mathrm{C} 2-\mathrm{H} 2$ & $0.98(2)$ & C16-H16B & $1.01(2)$ \\
\hline $\mathrm{C} 3-\mathrm{C} 4$ & $1.386(3)$ & $\mathrm{C} 17-\mathrm{C} 18$ & $1.519(3)$ \\
\hline $\mathrm{C} 3-\mathrm{H} 3$ & $0.99(2)$ & $\mathrm{C} 17-\mathrm{H} 17 \mathrm{~A}$ & $1.02(3)$ \\
\hline $\mathrm{C} 4-\mathrm{C} 5$ & $1.389(3)$ & C17-H17B & $1.01(2)$ \\
\hline $\mathrm{C} 4-\mathrm{H} 4$ & $0.94(2)$ & $\mathrm{C} 18-\mathrm{C} 19$ & $1.524(3)$ \\
\hline $\mathrm{C} 5-\mathrm{C} 6$ & $1.393(3)$ & $\mathrm{C} 18-\mathrm{H} 18 \mathrm{~A}$ & $1.00(3)$ \\
\hline $\mathrm{C} 5-\mathrm{H} 5$ & $0.96(2)$ & $\mathrm{C} 18-\mathrm{H} 18 \mathrm{~B}$ & $1.01(3)$ \\
\hline $\mathrm{C} 6-\mathrm{C} 7$ & $1.440(2)$ & $\mathrm{C} 19-\mathrm{C} 20$ & $1.518(3)$ \\
\hline $\mathrm{C} 7-\mathrm{C} 9$ & $1.350(3)$ & C19-H19A & $1.03(3)$ \\
\hline $\mathrm{C} 7-\mathrm{C} 8$ & $1.520(2)$ & C19-H19B & $1.04(2)$ \\
\hline $\mathrm{C} 9-\mathrm{C} 10$ & $1.437(3)$ & $\mathrm{C} 20-\mathrm{C} 21$ & $1.522(3)$ \\
\hline $\mathrm{C} 9-\mathrm{C} 11$ & $1.444(3)$ & $\mathrm{C} 20-\mathrm{H} 20 \mathrm{~A}$ & $1.02(3)$ \\
\hline $\mathrm{C} 12-\mathrm{C} 13$ & $1.526(2)$ & $\mathrm{C} 20-\mathrm{H} 20 \mathrm{~B}$ & $1.01(3)$ \\
\hline $\mathrm{C} 12-\mathrm{H} 12 \mathrm{~A}$ & $1.06(2)$ & $\mathrm{C} 21-\mathrm{H} 21 \mathrm{~A}$ & $1.01(3)$ \\
\hline $\mathrm{C} 12-\mathrm{H} 12 \mathrm{~B}$ & $1.01(2)$ & $\mathrm{C} 21-\mathrm{H} 21 \mathrm{~B}$ & $0.97(3)$ \\
\hline $\mathrm{C} 13-\mathrm{C} 14$ & $1.525(3)$ & $\mathrm{C} 21-\mathrm{H} 21 \mathrm{C}$ & $1.00(3)$ \\
\hline $\mathrm{C} 13-\mathrm{H} 13 \mathrm{~A}$ & $1.02(2)$ & & \\
\hline $\mathrm{C} 8-\mathrm{N} 1-\mathrm{C} 1$ & $110.66(14)$ & $\mathrm{C} 15-\mathrm{C} 14-\mathrm{H} 14 \mathrm{~A}$ & $109.4(13)$ \\
\hline $\mathrm{C} 8-\mathrm{N} 1-\mathrm{C} 12$ & $123.47(15)$ & $\mathrm{C} 13-\mathrm{C} 14-\mathrm{H} 14 \mathrm{~B}$ & $110.6(12)$ \\
\hline $\mathrm{C} 1-\mathrm{N} 1-\mathrm{C} 12$ & $125.69(15)$ & $\mathrm{C} 15-\mathrm{C} 14-\mathrm{H} 14 \mathrm{~B}$ & $109.3(13)$ \\
\hline $\mathrm{C} 2-\mathrm{C} 1-\mathrm{N} 1$ & $128.09(16)$ & $\mathrm{H} 14 \mathrm{~A}-\mathrm{C} 14-\mathrm{H} 14 \mathrm{~B}$ & $105.7(17)$ \\
\hline $\mathrm{C} 2-\mathrm{C} 1-\mathrm{C} 6$ & $121.89(16)$ & $\mathrm{C} 16-\mathrm{C} 15-\mathrm{C} 14$ & $114.37(16)$ \\
\hline $\mathrm{N} 1-\mathrm{C} 1-\mathrm{C} 6$ & $109.99(15)$ & $\mathrm{C} 16-\mathrm{C} 15-\mathrm{H} 15 \mathrm{~A}$ & $107.9(13)$ \\
\hline $\mathrm{C} 1-\mathrm{C} 2-\mathrm{C} 3$ & $117.34(17)$ & $\mathrm{C} 14-\mathrm{C} 15-\mathrm{H} 15 \mathrm{~A}$ & $109.6(13)$ \\
\hline $\mathrm{C} 1-\mathrm{C} 2-\mathrm{H} 2$ & $121.3(12)$ & $\mathrm{C} 16-\mathrm{C} 15-\mathrm{H} 15 \mathrm{~B}$ & $109.9(12)$ \\
\hline $\mathrm{C} 3-\mathrm{C} 2-\mathrm{H} 2$ & $121.3(12)$ & $\mathrm{C} 14-\mathrm{C} 15-\mathrm{H} 15 \mathrm{~B}$ & $110.8(12)$ \\
\hline $\mathrm{C} 4-\mathrm{C} 3-\mathrm{C} 2$ & $121.69(18)$ & $\mathrm{H} 15 \mathrm{~A}-\mathrm{C} 15-\mathrm{H} 15 \mathrm{~B}$ & $103.6(18)$ \\
\hline $\mathrm{C} 4-\mathrm{C} 3-\mathrm{H} 3$ & $119.3(13)$ & $\mathrm{C} 15-\mathrm{C} 16-\mathrm{C} 17$ & $113.24(16)$ \\
\hline $\mathrm{C} 2-\mathrm{C} 3-\mathrm{H} 3$ & $119.0(13)$ & $\mathrm{C} 15-\mathrm{C} 16-\mathrm{H} 16 \mathrm{~A}$ & $109.4(13)$ \\
\hline $\mathrm{C} 3-\mathrm{C} 4-\mathrm{C} 5$ & $120.72(18)$ & $\mathrm{C} 17-\mathrm{C} 16-\mathrm{H} 16 \mathrm{~A}$ & $109.8(14)$ \\
\hline $\mathrm{C} 3-\mathrm{C} 4-\mathrm{H} 4$ & $122.2(13)$ & $\mathrm{C} 15-\mathrm{C} 16-\mathrm{H} 16 \mathrm{~B}$ & $110.9(13)$ \\
\hline $\mathrm{C} 5-\mathrm{C} 4-\mathrm{H} 4$ & $117.1(13)$ & $\mathrm{C} 17-\mathrm{C} 16-\mathrm{H} 16 \mathrm{~B}$ & $108.5(13)$ \\
\hline
\end{tabular}




\begin{tabular}{|c|c|c|c|}
\hline $\mathrm{C} 4-\mathrm{C} 5-\mathrm{C} 6$ & $118.63(17)$ & $\mathrm{H} 16 \mathrm{~A}-\mathrm{C} 16-\mathrm{H} 16 \mathrm{~B}$ & $104.6(18)$ \\
\hline $\mathrm{C} 4-\mathrm{C} 5-\mathrm{H} 5$ & $119.9(13)$ & $\mathrm{C} 18-\mathrm{C} 17-\mathrm{C} 16$ & $113.37(16)$ \\
\hline $\mathrm{C} 6-\mathrm{C} 5-\mathrm{H} 5$ & $121.4(13)$ & $\mathrm{C} 18-\mathrm{C} 17-\mathrm{H} 17 \mathrm{~A}$ & $110.1(14)$ \\
\hline $\mathrm{C} 5-\mathrm{C} 6-\mathrm{C} 1$ & $119.70(17)$ & $\mathrm{C} 16-\mathrm{C} 17-\mathrm{H} 17 \mathrm{~A}$ & $108.2(14)$ \\
\hline $\mathrm{C} 5-\mathrm{C} 6-\mathrm{C} 7$ & $133.42(17)$ & $\mathrm{C} 18-\mathrm{C} 17-\mathrm{H} 17 \mathrm{~B}$ & $109.1(13)$ \\
\hline $\mathrm{C} 1-\mathrm{C} 6-\mathrm{C} 7$ & $106.87(15)$ & $\mathrm{C} 16-\mathrm{C} 17-\mathrm{H} 17 \mathrm{~B}$ & $108.2(13)$ \\
\hline $\mathrm{C} 9-\mathrm{C} 7-\mathrm{C} 6$ & $131.53(16)$ & $\mathrm{H} 17 \mathrm{~A}-\mathrm{C} 17-\mathrm{H} 17 \mathrm{~B}$ & $107.6(19)$ \\
\hline $\mathrm{C} 9-\mathrm{C} 7-\mathrm{C} 8$ & $121.89(16)$ & $\mathrm{C} 17-\mathrm{C} 18-\mathrm{C} 19$ & $114.73(17)$ \\
\hline $\mathrm{C} 6-\mathrm{C} 7-\mathrm{C} 8$ & $106.55(15)$ & $\mathrm{C} 17-\mathrm{C} 18-\mathrm{H} 18 \mathrm{~A}$ & $108.3(14)$ \\
\hline $\mathrm{O} 1-\mathrm{C} 8-\mathrm{N} 1$ & $127.13(16)$ & $\mathrm{C} 19-\mathrm{C} 18-\mathrm{H} 18 \mathrm{~A}$ & $109.8(14)$ \\
\hline $\mathrm{O} 1-\mathrm{C} 8-\mathrm{C} 7$ & $126.96(17)$ & $\mathrm{C} 17-\mathrm{C} 18-\mathrm{H} 18 \mathrm{~B}$ & $109.1(14)$ \\
\hline $\mathrm{N} 1-\mathrm{C} 8-\mathrm{C} 7$ & $105.90(15)$ & $\mathrm{C} 19-\mathrm{C} 18-\mathrm{H} 18 \mathrm{~B}$ & $108.1(15)$ \\
\hline $\mathrm{C} 7-\mathrm{C} 9-\mathrm{C} 10$ & $124.25(16)$ & $\mathrm{H} 18 \mathrm{~A}-\mathrm{C} 18-\mathrm{H} 18 \mathrm{~B}$ & $106(2)$ \\
\hline $\mathrm{C} 7-\mathrm{C} 9-\mathrm{C} 11$ & $121.24(18)$ & $\mathrm{C} 20-\mathrm{C} 19-\mathrm{C} 18$ & $113.30(17)$ \\
\hline $\mathrm{C} 10-\mathrm{C} 9-\mathrm{C} 11$ & $114.51(16)$ & $\mathrm{C} 20-\mathrm{C} 19-\mathrm{H} 19 \mathrm{~A}$ & $109.3(13)$ \\
\hline $\mathrm{N} 2-\mathrm{C} 10-\mathrm{C} 9$ & $173.7(2)$ & $\mathrm{C} 18-\mathrm{C} 19-\mathrm{H} 19 \mathrm{~A}$ & $110.1(14)$ \\
\hline $\mathrm{N} 3-\mathrm{C} 11-\mathrm{C} 9$ & $179.3(3)$ & $\mathrm{C} 20-\mathrm{C} 19-\mathrm{H} 19 \mathrm{~B}$ & $112.3(13)$ \\
\hline $\mathrm{N} 1-\mathrm{C} 12-\mathrm{C} 13$ & $113.70(15)$ & $\mathrm{C} 18-\mathrm{C} 19-\mathrm{H} 19 \mathrm{~B}$ & $107.9(12)$ \\
\hline $\mathrm{N} 1-\mathrm{C} 12-\mathrm{H} 12 \mathrm{~A}$ & $106.2(12)$ & $\mathrm{H} 19 \mathrm{~A}-\mathrm{C} 19-\mathrm{H} 19 \mathrm{~B}$ & $103.6(18)$ \\
\hline $\mathrm{C} 13-\mathrm{C} 12-\mathrm{H} 12 \mathrm{~A}$ & $108.8(11)$ & $\mathrm{C} 19-\mathrm{C} 20-\mathrm{C} 21$ & $113.11(19)$ \\
\hline $\mathrm{N} 1-\mathrm{C} 12-\mathrm{H} 12 \mathrm{~B}$ & $106.6(12)$ & $\mathrm{C} 19-\mathrm{C} 20-\mathrm{H} 20 \mathrm{~A}$ & $109.7(14)$ \\
\hline $\mathrm{C} 13-\mathrm{C} 12-\mathrm{H} 12 \mathrm{~B}$ & $110.0(12)$ & $\mathrm{C} 21-\mathrm{C} 20-\mathrm{H} 20 \mathrm{~A}$ & $110.3(14)$ \\
\hline $\mathrm{H} 12 \mathrm{~A}-\mathrm{C} 12-\mathrm{H} 12 \mathrm{~B}$ & $111.6(17)$ & $\mathrm{C} 19-\mathrm{C} 20-\mathrm{H} 20 \mathrm{~B}$ & $106.0(13)$ \\
\hline $\mathrm{C} 14-\mathrm{C} 13-\mathrm{C} 12$ & $114.63(16)$ & $\mathrm{C} 21-\mathrm{C} 20-\mathrm{H} 20 \mathrm{~B}$ & $110.7(14)$ \\
\hline $\mathrm{C} 14-\mathrm{C} 13-\mathrm{H} 13 \mathrm{~A}$ & $108.8(12)$ & $\mathrm{H} 20 \mathrm{~A}-\mathrm{C} 20-\mathrm{H} 20 \mathrm{~B}$ & $107(2)$ \\
\hline $\mathrm{C} 12-\mathrm{C} 13-\mathrm{H} 13 \mathrm{~A}$ & $105.0(13)$ & $\mathrm{C} 20-\mathrm{C} 21-\mathrm{H} 21 \mathrm{~A}$ & $110.0(15)$ \\
\hline $\mathrm{C} 14-\mathrm{C} 13-\mathrm{H} 13 \mathrm{~B}$ & $112.0(12)$ & $\mathrm{C} 20-\mathrm{C} 21-\mathrm{H} 21 \mathrm{~B}$ & $112.0(18)$ \\
\hline $\mathrm{C} 12-\mathrm{C} 13-\mathrm{H} 13 \mathrm{~B}$ & $108.1(11)$ & $\mathrm{H} 21 \mathrm{~A}-\mathrm{C} 21-\mathrm{H} 21 \mathrm{~B}$ & $108(2)$ \\
\hline $\mathrm{H} 13 \mathrm{~A}-\mathrm{C} 13-\mathrm{H} 13 \mathrm{~B}$ & $108.0(17)$ & $\mathrm{C} 20-\mathrm{C} 21-\mathrm{H} 21 \mathrm{C}$ & $109.0(16)$ \\
\hline $\mathrm{C} 13-\mathrm{C} 14-\mathrm{C} 15$ & $111.49(16)$ & $\mathrm{H} 21 \mathrm{~A}-\mathrm{C} 21-\mathrm{H} 21 \mathrm{C}$ & $110(2)$ \\
\hline $\mathrm{C} 13-\mathrm{C} 14-\mathrm{H} 14 \mathrm{~A}$ & $110.3(13)$ & $\mathrm{H} 21 \mathrm{~B}-\mathrm{C} 21-\mathrm{H} 21 \mathrm{C}$ & $107(2)$ \\
\hline $\mathrm{C} 8-\mathrm{N} 1-\mathrm{C} 1-\mathrm{C} 2$ & $176.31(17)$ & $\mathrm{C} 1-\mathrm{N} 1-\mathrm{C} 8-\mathrm{C} 7$ & 1.67 (19) \\
\hline $\mathrm{C} 12-\mathrm{N} 1-\mathrm{C} 1-\mathrm{C} 2$ & $1.2(3)$ & $\mathrm{C} 12-\mathrm{N} 1-\mathrm{C} 8-\mathrm{C} 7$ & $176.95(15)$ \\
\hline $\mathrm{C} 8-\mathrm{N} 1-\mathrm{C} 1-\mathrm{C} 6$ & $-1.9(2)$ & $\mathrm{C} 9-\mathrm{C} 7-\mathrm{C} 8-\mathrm{O} 1$ & $-0.6(3)$ \\
\hline $\mathrm{C} 12-\mathrm{N} 1-\mathrm{C} 1-\mathrm{C} 6$ & $-177.06(16)$ & $\mathrm{C} 6-\mathrm{C} 7-\mathrm{C} 8-\mathrm{O} 1$ & $177.92(18)$ \\
\hline $\mathrm{N} 1-\mathrm{C} 1-\mathrm{C} 2-\mathrm{C} 3$ & $179.73(17)$ & $\mathrm{C} 9-\mathrm{C} 7-\mathrm{C} 8-\mathrm{N} 1$ & $-179.39(17)$ \\
\hline $\mathrm{C} 6-\mathrm{C} 1-\mathrm{C} 2-\mathrm{C} 3$ & $-2.3(3)$ & $\mathrm{C} 6-\mathrm{C} 7-\mathrm{C} 8-\mathrm{N} 1$ & $-0.88(19)$ \\
\hline $\mathrm{C} 1-\mathrm{C} 2-\mathrm{C} 3-\mathrm{C} 4$ & $1.1(3)$ & $\mathrm{C} 6-\mathrm{C} 7-\mathrm{C} 9-\mathrm{C} 10$ & $-177.85(18)$ \\
\hline $\mathrm{C} 2-\mathrm{C} 3-\mathrm{C} 4-\mathrm{C} 5$ & $0.7(3)$ & $\mathrm{C} 8-\mathrm{C} 7-\mathrm{C} 9-\mathrm{C} 10$ & $0.2(3)$ \\
\hline $\mathrm{C} 3-\mathrm{C} 4-\mathrm{C} 5-\mathrm{C} 6$ & $-1.4(3)$ & $\mathrm{C} 6-\mathrm{C} 7-\mathrm{C} 9-\mathrm{C} 11$ & $1.6(3)$ \\
\hline $\mathrm{C} 4-\mathrm{C} 5-\mathrm{C} 6-\mathrm{C} 1$ & $0.3(3)$ & $\mathrm{C} 8-\mathrm{C} 7-\mathrm{C} 9-\mathrm{C} 11$ & $179.71(17)$ \\
\hline $\mathrm{C} 4-\mathrm{C} 5-\mathrm{C} 6-\mathrm{C} 7$ & $178.57(19)$ & $\mathrm{C} 8-\mathrm{N} 1-\mathrm{C} 12-\mathrm{C} 13$ & $112.3(2)$ \\
\hline $\mathrm{C} 2-\mathrm{C} 1-\mathrm{C} 6-\mathrm{C} 5$ & $1.6(3)$ & $\mathrm{C} 1-\mathrm{N} 1-\mathrm{C} 12-\mathrm{C} 13$ & $-73.1(2)$ \\
\hline $\mathrm{N} 1-\mathrm{C} 1-\mathrm{C} 6-\mathrm{C} 5$ & $179.93(15)$ & $\mathrm{N} 1-\mathrm{C} 12-\mathrm{C} 13-\mathrm{C} 14$ & $-62.1(2)$ \\
\hline $\mathrm{C} 2-\mathrm{C} 1-\mathrm{C} 6-\mathrm{C} 7$ & $-177.09(16)$ & $\mathrm{C} 12-\mathrm{C} 13-\mathrm{C} 14-\mathrm{C} 15$ & $-173.98(15)$ \\
\hline $\mathrm{N} 1-\mathrm{C} 1-\mathrm{C} 6-\mathrm{C} 7$ & $1.25(19)$ & $\mathrm{C} 13-\mathrm{C} 14-\mathrm{C} 15-\mathrm{C} 16$ & $179.80(16)$ \\
\hline $\mathrm{C} 5-\mathrm{C} 6-\mathrm{C} 7-\mathrm{C} 9$ & $-0.3(3)$ & $\mathrm{C} 14-\mathrm{C} 15-\mathrm{C} 16-\mathrm{C} 17$ & $-178.34(16)$ \\
\hline
\end{tabular}


$\mathrm{C} 1-\mathrm{C} 6-\mathrm{C} 7-\mathrm{C} 9$
$\mathrm{C} 5-\mathrm{C} 6-\mathrm{C} 7-\mathrm{C} 8$
$\mathrm{C} 1-\mathrm{C} 6-\mathrm{C} 7-\mathrm{C} 8$
$\mathrm{C} 1-\mathrm{N} 1-\mathrm{C} 8-\mathrm{O} 1$
$\mathrm{C} 12-\mathrm{N} 1-\mathrm{C} 8-\mathrm{O} 1$

178.09 (19)

$-178.65(19)$

$-0.23(18)$

$-177.13(18)$

$-1.8(3)$

$\begin{array}{ll}\mathrm{C} 15-\mathrm{C} 16-\mathrm{C} 17-\mathrm{C} 18 & -179.93(17) \\ \mathrm{C} 16-\mathrm{C} 17-\mathrm{C} 18-\mathrm{C} 19 & -179.68(17) \\ \mathrm{C} 17-\mathrm{C} 18-\mathrm{C} 19-\mathrm{C} 20 & -179.44(18) \\ \mathrm{C} 18-\mathrm{C} 19-\mathrm{C} 20-\mathrm{C} 21 & 178.45(19)\end{array}$

$\mathrm{C} 15-\mathrm{C} 16-\mathrm{C} 17-\mathrm{C} 18$

$-179.93(17)$

$-179.44(18)$ 